\title{
Correction to: Scene Understanding in Night-Time Using SSAN Dataset
}

R Anandha Murugan, B Sathyabama,

Sam Joshuva Paul Jeevan Shapher, Suryakiran Sureshkumar, and Nighil Krishna Rajaguru

\section{Correction to:}

Chapter "Scene Understanding in Night-Time Using SSAN

Dataset" in: R. V. Babu et al. (Eds.): Computer Vision,

Pattern Recognition, Image Processing, and Graphics,

CCIS 1249, https://doi.org/10.1007/978-981-15-8697-2_52

The original version of this chapter was revised. The names of the authors "Sam Joshuva Paul Jeevan Shapher, Suryakiran Sureshkumar and Nighil Krishna Rajaguru" were corrected. 